\title{
SPACECRAFT ATTITUDE FAULT TOLERANT CONTROL BASED ON MULTI-OBJECTIVE OPTIMIZATION
}

\author{
Rouzbeh Moradi, Alireza Alikhani \\ Aerospace Research Institute, Ministry of Science, Research and Technology, Tehran, Iran \\ e-mail: aalikhani@ari.ac.ir \\ Mohsen FATHi JeGARKANDi \\ Sharif University of Technology, Department of Aerospace Engineering, Tehran, Iran
}

\begin{abstract}
An active fault tolerant controller is designed for the attitude control of a faulty spacecraft. Feedback linearization and Lyapunov's direct method are used to solve angular velocity equations and to ensure convergence of the system outputs to reference inputs, respectively. In order to ensure finite time convergence, final time constraints are proposed. Three constructive objective functions are considered as performance measures and optimized using multi-objective optimization. The results show that the outputs converge to the reference attitudes, even for severe actuator faults/failures.
\end{abstract}

Keywords: active fault tolerant control, spacecraft attitude control, finite time convergence, Pareto optimal set

\section{Introduction}

It may be difficult to control a complex and faulty system with satisfactory performance using conventional state-feedback control (Zhang and Jiang, 2008; Fekih, 2014). Fault tolerant control (FTC) is a field that considers controlling faulty systems (Linlin, 2016). There are numerous books, papers and theses that have studied this challenging problem, e.g., Blanke et al. (2006), Lunze and Richter (2008), Noura et al. (2009), Witczak (2014), Jiang and Yu (2012), Alikhani (2017), Hu et al. (2017, 2018). According to the literature, FTC is divided into two main subsets: Passive FTC (PFTC) and Active FTC (AFTC) (Jiang and Yu, 2012). When designing PFTC, the main focus is on robustness of the closed-loop system, while AFTC considers performance measures as well. PFTC has a fixed structure, while AFTC has a flexible structure. This makes AFTC more flexible toward faults and, therefore, capable of dealing with a broader range of unanticipated faults/failures (Jiang and $\mathrm{Yu}, 2012$ ).

According to Tafazoli (2008), actuator fault/failure has a destructive effect on the Attitude Control System (ACS) performance. According to this reference, nearly $45 \%$ of ACS failures have occurred due to actuator faults. Therefore, actuator fault/failure is an important problem that deserves special attention when designing an ACS system. Recently, a review paper has performed a literature study on fault-tolerant ACS (Yin et al. (2016)). Assuming a fixed controller structure (as common with PFTCs), full-actuation and sufficient actuator redundancy have been the main underlying assumptions of the previous works in this area.

Although it is possible to consider enough actuator redundancy to make sure that the faulty system has enough actuation, more redundancy means more weight and cost (not desirable features, especially for space missions). It may be possible to use controller switching in cases of actuator failure (Jiang and Zhang, 2006). However, this approach requires an "engineering understanding" of the faulty system performance, especially when a combination of faults/failures occurs in the actuation system (Benosman and Lum, 2009). 
In this paper, a unified AFTC framework is proposed for the spacecraft attitude control problem. This approach reduces the need for actuator redundancy and does not require controller switching. Lyapunov's direct method, a theorem and several constraints are used to ensure finite time convergence of Euler angles to the reference attitude. Three objective functions are considered as performance measures and optimized using multi-objective optimization. The proposed controller shows an acceptable performance, especially for severe actuator failures.

The rest of the paper consists of the following sections: Section 2 discusses dynamics of the spacecraft. Dynamic equations and fault scenario are the topics of this Section. Section 3 deals with the design of the proposed active fault tolerant controller, where its subsystems are analyzed in detail and a theorem is presented. In order to evaluate the controller performance, several simulations are presented and analyzed in Section 4. Finally, a conclusion is presented to sum-up the paper.

\section{Dynamics of spacecraft and fault scenario}

\subsection{Dynamic equations}

The rigid body spacecraft rotational dynamics in the principal coordinate system is described by the following equations (Zipfel, 2007)

$$
\dot{\mathbf{q}}=-\frac{1}{2} \Omega \mathbf{q} \quad \dot{\boldsymbol{\omega}}=-\mathbf{J}^{-1}(\boldsymbol{\omega} \times \mathbf{J} \boldsymbol{\omega})+\mathbf{J}^{-1} \mathbf{u}
$$

where: $\mathbf{q}$ - quaternion vector, $\boldsymbol{\omega}$ - angular velocity vector, $\mathbf{J}$ - moment of inertia matrix, $\mathbf{u}$ - control moments acting on the spacecraft

$$
\begin{aligned}
& \mathbf{q}^{\mathrm{T}}=q_{0}+\mathbf{i} q_{1}+\mathbf{j} q_{2}+\mathbf{k} q_{3} \\
& \mathbf{J}=\left[\begin{array}{ccc}
J_{1} & 0 & 0 \\
0 & J_{2} & 0 \\
0 & 0 & J_{3}
\end{array}\right] \quad \boldsymbol{\omega ^ { \mathrm { T } } = [ \omega _ { 1 } , \omega _ { 2 } , \omega _ { 3 } ]} \quad \mathbf{u}^{\mathrm{T}}=\left[u_{1}, u_{2}, u_{3}\right] \\
& \mathbf{\Omega}=\left[\begin{array}{cccc}
0 & \omega_{1} & \omega_{2} & \omega_{3} \\
-\omega_{1} & 0 & -\omega_{3} & \omega_{2} \\
-\omega_{2} & \omega_{3} & 0 & -\omega_{1} \\
-\omega_{3} & -\omega_{2} & \omega_{1} & 0
\end{array}\right]
\end{aligned}
$$

Equations (2.1) are expressed in body coordinates with respect to the inertial reference frame (Zipfel, 2007).

The reason for using quaternion-based equations is to prevent singularity. But the Euler angles have the advantage over quaternions being more physically sensible. Therefore, Eqs. (2.3) are used to convert Euler angles $(\phi, \theta, \psi)$ (with the sequence $\psi-\theta-\phi)$ to quaternions (Zipfel, 2007)

$$
\begin{aligned}
& q_{0}=\cos \frac{\psi}{2} \cos \frac{\theta}{2} \cos \frac{\phi}{2}+\sin \frac{\psi}{2} \sin \frac{\theta}{2} \sin \frac{\phi}{2} \\
& q_{1}=\cos \frac{\psi}{2} \cos \frac{\theta}{2} \sin \frac{\phi}{2}-\sin \frac{\psi}{2} \sin \frac{\theta}{2} \cos \frac{\phi}{2} \\
& q_{2}=\cos \frac{\psi}{2} \sin \frac{\theta}{2} \cos \frac{\phi}{2}+\sin \frac{\psi}{2} \cos \frac{\theta}{2} \sin \frac{\phi}{2} \\
& q_{3}=\sin \frac{\psi}{2} \cos \frac{\theta}{2} \cos \frac{\phi}{2}-\cos \frac{\psi}{2} \sin \frac{\theta}{2} \sin \frac{\phi}{2}
\end{aligned}
$$

In order to return the results in the form of Euler angles, the following expressions will be used (Zipfel, 2007)

$$
\begin{aligned}
\tan \psi & =\frac{2\left(q_{1} q_{2}+q_{0} q_{3}\right)}{q_{0}^{2}+q_{1}^{2}-q_{2}^{2}-q_{3}^{2}} \quad \sin \theta=-2\left(q_{1} q_{3}-q_{0} q_{2}\right) \\
\tan \phi & =\frac{2\left(q_{2} q_{3}+q_{0} q_{1}\right)}{q_{0}^{2}-q_{1}^{2}-q_{2}^{2}+q_{3}^{2}}
\end{aligned}
$$


According to the separation theorem, if the center of mass is selected as the reference point, the rotational and translational dynamics will be separated (Zipfel, 2007). On the other hand, if the spacecraft is orbiting the earth at high altitude, the aerodynamic effects can be neglected. Therefore, it is assumed that the translational and rotational dynamics are separated.

\subsection{Fault scenario}

Several factors leading to ACS failure (Tafazoli, 2008) are presented in Fig. 1.
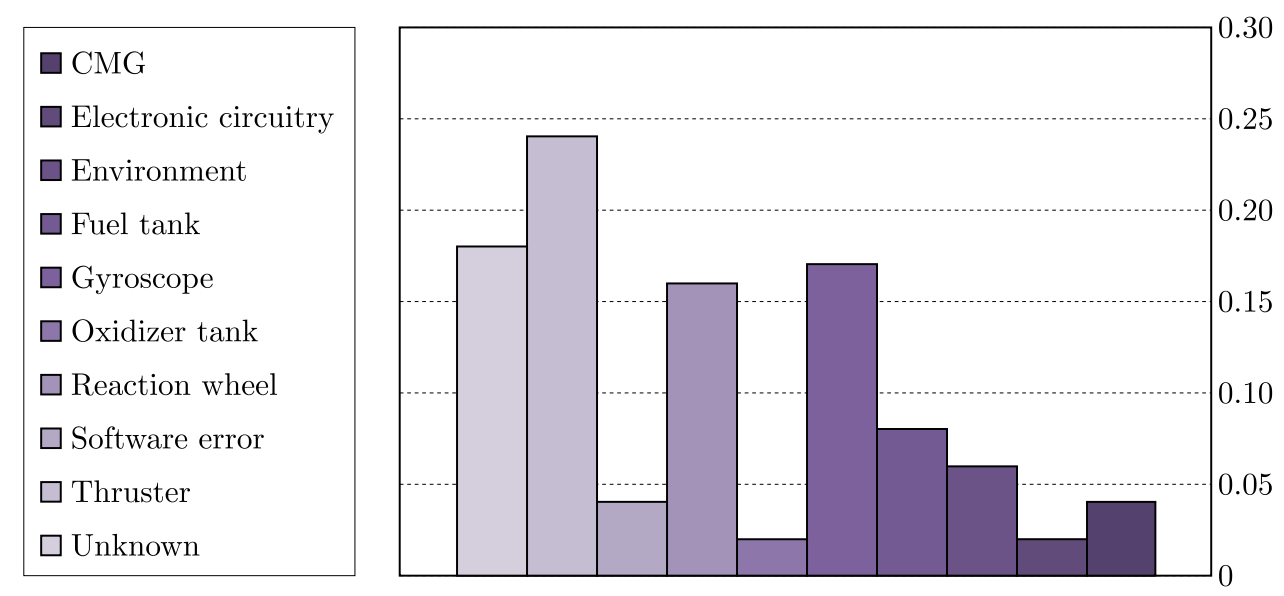

Fig. 1. Contributing factors involved in ACS failure (Tafazoli, 2008)

This figure shows that almost $45 \%$ of ACS failure is due to actuator faults (Control moment gyroscope (CMG), reaction wheel, and thrusters). Therefore, the actuator fault is an important source of ACS failure.

It is assumed that the post-fault actuator performance region is given as

$$
\operatorname{sat}_{n}\left(u_{i}\right)= \begin{cases}u_{i} & \text { if }-u_{\max , n} \leqslant u_{i} \leqslant u_{\max , n} \\ u_{\max , n} & \text { if } u_{i}>u_{\max , n} \\ -u_{\max , n} & \text { if } u_{i}<-u_{\max , n}\end{cases}
$$

where $u_{\max , n}$ is the maximum control moment that can be produced after the occurrence of a fault in the actuators. The relation between $u_{\max , n}$ and $u_{\max }$ (pre-fault maximum control moment) is given by

$$
u_{\max , n}=a u_{\max } \quad 0 \leqslant a \leqslant 1
$$

For a healthy and failed actuator, $a$ is 1 and 0, respectively (Miksch and Gambier, 2011).

\section{Active fault tolerant controller design}

A block diagram of the closed-loop control system is shown in Fig. 2.

The fault detection and diagnosis (FDD) unit detects the plant of the post-fault system and the reconfiguration mechanism receives this information immediately. Then, the reconfiguration mechanism produces modified reference inputs and adjusts controller parameters. The objective of this reconfiguration is to satisfy the post-fault requirements of the mission. It should be noted that angular velocity feedback is sufficient to close the control loop, Eq. (3.1). 


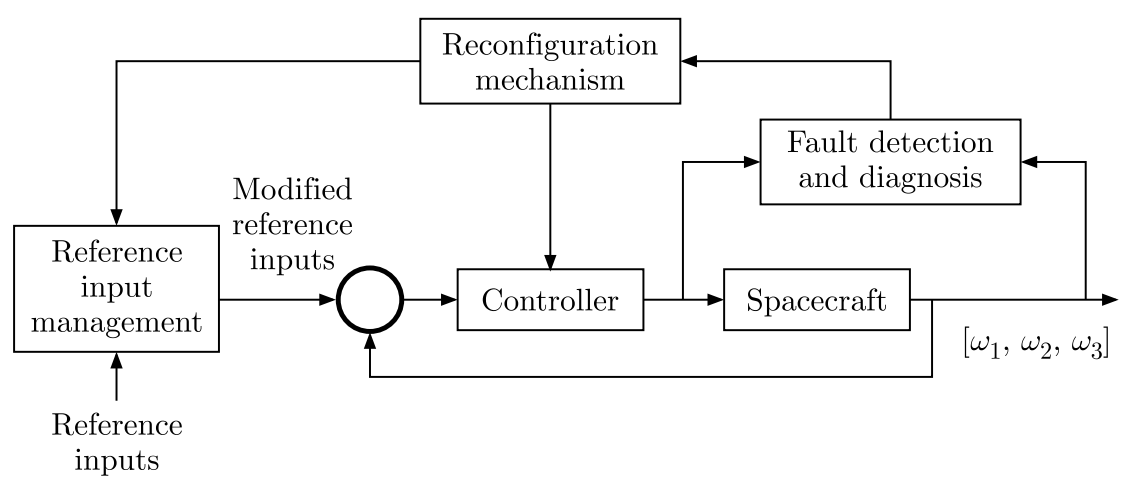

Fig. 2. Block diagram of the closed-loop control system

Note 1: The pre-fault (healthy) system should be able to track the reference inputs, e.g. exponentially or asymptotically, posed by the mission requirements. However, after the occurrence of a severe fault/failure, such a requirement may not be satisfied. Therefore, modified reference inputs are designed such that the faulty system response (in terms of the Euler angles) would converge to the reference inputs in a finite time.

\subsection{Reference input management and controller}

Feedback control laws are considered as

$$
\mathbf{u}=-\mathbf{J K}\left(\boldsymbol{\omega}-\boldsymbol{\omega}_{m}\right)+\mathbf{J} \dot{\boldsymbol{\omega}}_{m}+\boldsymbol{\omega} \times \mathbf{J} \boldsymbol{\omega}
$$

where

$$
\mathbf{K}=\left[\begin{array}{ccc}
k_{1} & 0 & 0 \\
0 & k_{2} & 0 \\
0 & 0 & k_{3}
\end{array}\right] \quad k_{1}, k_{2}, k_{3} \in R^{+}
$$

where $k_{1}, k_{2}, k_{3}$ are the controller coefficients and $\boldsymbol{\omega}_{m}$ is the modified reference input vector. It can be easily shown that considering Eqs. $(2.1)_{2}$ and (3.1), the closed-loop system for angular velocities will be

$$
\dot{\boldsymbol{\omega}}_{e}=-\mathbf{K} \boldsymbol{\omega}_{e} \quad \boldsymbol{\omega}_{e}=\boldsymbol{\omega}-\boldsymbol{\omega}_{m}
$$

showing exponential convergence of $\boldsymbol{\omega}_{e}$ to zero, or equivalently, $\boldsymbol{\omega}$ to $\boldsymbol{\omega}_{m}$. According to Eq. $(2.1)_{1}$, the spacecraft attitude is a function of the angular velocity. Therefore, in order to adjust the attitude, the spacecraft should perform the appropriate angular velocity maneuver. In other words, by producing an appropriate $\boldsymbol{\omega}_{m}$, the spacecraft will be able to perform the corresponding attitude maneuvers.

In order to ensure exponential convergence of the Euler angles $\phi, \theta, \psi$ to the modified reference inputs $\left(\phi_{m}, \theta_{m}, \psi_{m}\right)$, the following quadratic Lyapunov function is considered

$$
V=e_{\phi}^{2}+e_{\theta}^{2}+e_{\psi}^{2}
$$

where

$$
\begin{array}{ll}
e_{\phi}=\phi-\phi_{m} & \phi_{m}=\phi_{r}+\phi_{m_{0}} \\
e_{\theta}=\theta-\theta_{m} & \theta_{m}=\theta_{r}+\theta_{m_{0}} \\
e_{\psi}=\psi-\psi_{m} & \psi_{m}=\psi_{r}+\psi_{m_{0}}
\end{array}
$$


The three variables $\phi_{m_{0}}, \theta_{m_{0}}, \psi_{m_{0}}$ are modified by the reconfiguration mechanism. $\phi_{r}, \theta_{r}, \psi_{r}$ are the three reference inputs that the post-fault system outputs $(\phi, \theta, \psi)$ should converge to.

According to Lyapunov's second method for stability (Khalil, 2014), if the time derivative of Lyapunov function (3.3) satisfies the equation

$$
\dot{V}=-k V \quad k>0
$$

the conditions of Lyapunov's second method for stability will be satisfied and the post-fault system outputs $(\phi, \theta, \psi)$ will converge to the modified reference inputs $\left(\phi_{m}, \theta_{m}, \psi_{m}\right)$ with an exponential rate $k$.

The numerical derivative of Lyapunov's function may not be accurate. Therefore, Eq. (3.6) will be used instead of Eq. (3.5)

$$
V=V(0) \mathrm{e}^{-k t} \quad k>0
$$

Equation (3.6) is the solution to Eq. (3.5). To make sure that the Lyapunov function and its time derivative become zero at the final time (finite time convergence), the following final time $t_{f}$ constraint is considered

$$
V\left(t=t_{f}\right)=0
$$

\subsection{Reconfiguration mechanism}

The reconfiguration mechanism uses constrained optimization to solve the optimal control problem defined as follows.

Determine the modified reference inputs, controller parameters and the parameter $k$ to minimize the following performance measures, simultaneously

$$
\begin{aligned}
& P M_{1}=\phi_{m_{0}}^{2}+\theta_{m_{0}}^{2}+\psi_{m_{0}}^{2} \quad \text { at } \quad t=t_{f} \\
& P M_{2}=\dot{\phi}_{m_{0}}^{2}+\dot{\theta}_{m_{0}}^{2}+\dot{\psi}_{m_{0}}^{2} \quad \text { at } \quad t=t_{f} \\
& P M_{3}=\int_{t=t_{\text {fault }}}^{t=t_{f}}\left(u_{1}^{2}+u_{2}^{2}+u_{3}^{2}\right) d t
\end{aligned}
$$

subject to the following nonlinear constraints:

1) Dynamics and actuation: Eqs. (2.1)-(2.6)

2) Stability and control: Eqs. (3.1)-(3.4), (3.6) and (3.7).

A block diagram of the reconfiguration mechanism is shown in Fig. 3.

The numbers show the order of execution in the optimization. In the following, each block is described in detail:

1 - The optimization parameters (controller coefficients, parameter $k$, and modified reference inputs) are determined using cubic interpolating splines (Boor, 1978), in the fixed intervals of time $\delta t^{1}$.

2 - Splines are constructed.

3 - The closed-loop system is simulated from $t_{\text {fault }}$ to $t_{f}$, based on the information provided by the FDD.

\footnotetext{
${ }^{1} \mathrm{~A}$ large/small value of this parameter reduces/increases the optimization time and reduces/increases the chances of obtaining the best solution.
} 


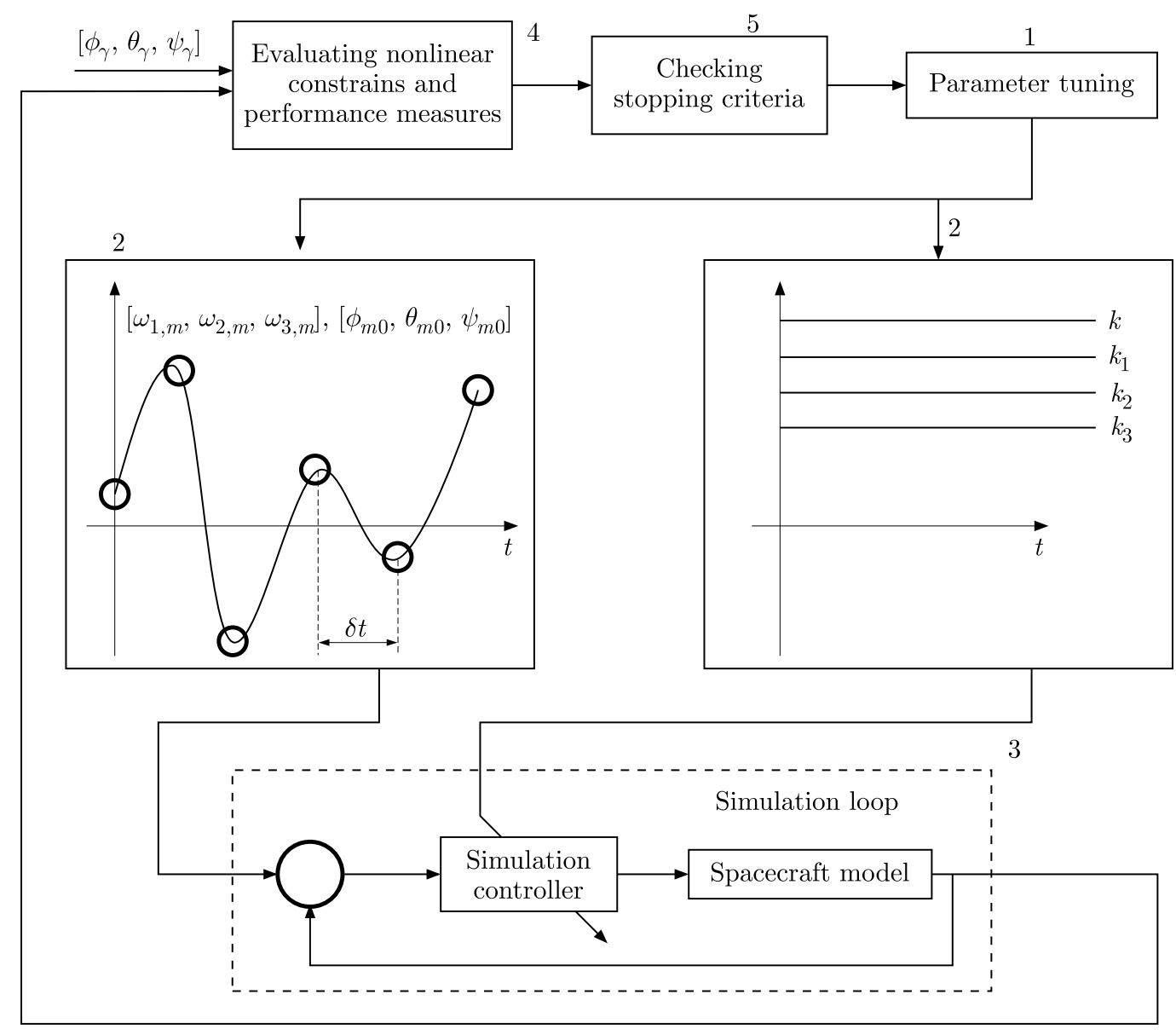

Fig. 3. Block diagram of the reconfiguration mechanism

4 - Performance measures and constraints are evaluated.

5 - Stopping criteria, e.g. the number of simulations is checked. If the stopping criteria are satisfied, the process finishes and, otherwise, continues.

Note 2: Due to the presence of three performance measures, the optimization problem is multi-objective. Physically, these performance measures are competitive, i.e. they increase in opposite directions. The result of this kind of optimization problem is a Pareto front, i.e. a set of optimal solutions (a single solution does not exist) (Marler and Arora, 2004).

\subsection{Theorem: Convergence of Euler angles to reference inputs}

Under the given constraints, minimizing $P M_{1}$ and $P M_{2}$ will lead to the convergence of Euler angles to the reference inputs.

$\underline{\text { Proof }}$

In order to prove this theorem, it is sufficient to prove that

$$
\lim _{t \rightarrow t_{f}} \mathbf{r}_{m_{0}}(t)=\mathbf{0} \quad \mathbf{r}_{m_{0}}=\left[\phi_{m_{0}}, \theta_{m_{0}}, \psi_{m_{0}}\right]
$$

Consider the following equation

$$
\dot{\mathbf{r}}_{m_{0}}\left(t_{f}\right)=\lim _{\Delta t \rightarrow 0} \frac{\mathbf{r}_{m_{0}}\left(t_{f}\right)-\mathbf{r}_{m_{0}}\left(t_{f}-\Delta t\right)}{\Delta t}
$$


where $\Delta t$ is defined as follows

$$
\Delta t=t_{f}-t
$$

According to Eqs. (3.8) $)_{1,2}$, the minimum value of $P M_{1}$ and $P M_{2}$ is zero, where equivalently

$$
\begin{aligned}
& \phi_{m_{0}}\left(t_{f}\right)=\theta_{m_{0}}\left(t_{f}\right)=\psi_{m_{0}}\left(t_{f}\right)=0 \rightarrow \mathbf{r}_{m_{0}}\left(t_{f}\right)=\mathbf{0} \\
& \dot{\phi}_{m_{0}}\left(t_{f}\right)=\dot{\theta}_{m_{0}}\left(t_{f}\right)=\dot{\psi}_{m_{0}}\left(t_{f}\right)=0 \rightarrow \dot{\mathbf{r}}_{m_{0}}\left(t_{f}\right)=\mathbf{0}
\end{aligned}
$$

Considering Eqs. (3.10) and (3.11) will result in

$$
\dot{\mathbf{r}}_{m_{0}}\left(t_{f}\right)=\lim _{\Delta t \rightarrow 0} \frac{\mathbf{r}_{m_{0}}\left(t_{f}-\Delta t\right)}{\Delta t}=\mathbf{0}
$$

Substituting Eq. (3.11) into Eq. (3.13) will result in

$$
\lim _{t \rightarrow t_{f}} \frac{\mathbf{r}_{m_{0}}\left(t_{f}-t_{f}+t\right)}{t_{f}-t}=\mathbf{0}
$$

In order for Eq. (3.14) to be satisfied, the numerator should converge more rapidly than the denominator to zero, therefore

$$
\lim _{t \rightarrow t_{f}} \mathbf{r}_{m_{0}}(t)=\mathbf{0}
$$

This statement shows that if $P M_{1}$ and $P M_{2}$ are minimized, $\mathbf{r}_{m_{0}}$ will converge to zero as $t$ reaches the final time. Therefore, according to Eq. (3.4)

$$
\begin{aligned}
& \lim _{t \rightarrow t_{f}} \phi_{m}=\lim _{t \rightarrow t_{f}} \phi_{r}+\lim _{t \rightarrow t_{f}} \phi_{m_{0}}=\lim _{t \rightarrow t_{f}} \phi_{r} \\
& \lim _{t \rightarrow t_{f}} \theta_{m}=\lim _{t \rightarrow t_{f}} \theta_{r}+\lim _{t \rightarrow t_{f}} \theta_{m_{0}}=\lim _{t \rightarrow t_{f}} \theta_{r} \\
& \lim _{t \rightarrow t_{f}} \psi_{m}=\lim _{t \rightarrow t_{f}} \psi_{r}+\lim _{t \rightarrow t_{f}} \psi_{m_{0}}=\lim _{t \rightarrow t_{f}} \psi_{r}
\end{aligned}
$$

Equations (3.16) show the convergence of the modified Euler angles to the reference Euler angles. Since it was shown that the Euler angles $(\phi, \theta, \psi)$ would converge to the modified Euler angles $\left(\phi_{m}, \theta_{m}, \psi_{m}\right)$ with an exponential rate $k$, it can be concluded that the Euler angles will converge to the reference inputs, provided that the given constraints are satisfied.

This completes the proof of the theorem.

\section{Simulation}

In order to show the performance of the proposed controller, several simulations have been carried out, and the results are illustrated in this Section. The initial conditions are considered to be: $\phi(0)=15, \theta(0)=10$ and $\psi(0)=-5$ all in deg.

It is assumed that the initial conditions for angular velocities are small. This assumption can be easily satisfied using angular velocity stabilization methods (Moradi et al., 2017). The principal moments of inertia are considered to be: $J_{1}=449.5, J_{2}=264.6, J_{3}=312.5$ all in $\mathrm{kg} \mathrm{m}^{2}$. The spacecraft physical parameters are directly selected from the data provided by Wang et al. (2013).

The lower and upper bounds and initial values for the optimization variables are given in Table 1.

The parameters in Table 1 are adjusted by the reconfiguration mechanism. For example, after the occurrence of a fault/failure, the reconfiguration mechanism decides to modify $\boldsymbol{\omega}_{m}(t=0)$. 
Table 1. Lower and upper bounds and initial values for the optimization variables

\begin{tabular}{|c|c|c|}
\hline Variables & Bound & Initial value \\
\hline \hline $\mathbf{r}_{m_{0}}$ & $\pm 30 \mathrm{deg}$ & $0 \mathrm{deg}$ \\
\hline$\dot{\boldsymbol{\omega}}_{m}$ & $\pm 10 \mathrm{deg} / \mathrm{s}^{2}$ & $0 \mathrm{deg} / \mathrm{s}^{2}$ \\
\hline $\boldsymbol{\omega}_{m}(t=0)$ & $\pm 10 \mathrm{deg} / \mathrm{s}$ & $0 \mathrm{deg} / \mathrm{s}$ \\
\hline$k_{1}, k_{2}, k_{3}$ & {$[0.5,2]$} & 1 \\
\hline$k$ & {$[0.5,10]$} & 5 \\
\hline
\end{tabular}

This parameter (and maybe the other parameters in Table 1) changes until the constraints and stopping criteria are satisfied. This process occurs as shown in Fig. 3. Instead of $\boldsymbol{\omega}_{m}, \dot{\boldsymbol{\omega}}_{m}$ and $\boldsymbol{\omega}_{m}(t=0)$ have been chosen as the optimization variables (to avoid the difficulties with numerical derivative) and $\delta t$ is taken as $1 \mathrm{~s}$ (refer to Fig. 3).

The reference inputs are given as (in deg)

$$
\phi_{r}=0.1 t \quad \theta_{r}=0.1 t \quad \psi_{r}=0.1 t
$$

These references are specified according to the mission specifications. Such specifications can be varied form mission to mission.

Table 2 illustrates the actuator effectiveness coefficients, corresponding to three cases.

Table 2. Actuator effectiveness coefficients for three cases

\begin{tabular}{|l|c|c|c|}
\hline & $a_{1}$ & $a_{2}$ & $a_{3}$ \\
\hline \hline First case (without fault) & 1 & 1 & 1 \\
\hline Second case & 1 & 1 & 0.1 \\
\hline Third case (actuator failure) & 1 & 1 & 0 \\
\hline
\end{tabular}

According to Table 2, in the first case, all of the actuators are healthy. In the second case, the actuator fault leads to $90 \%$ degradation in the moment that can be produced about the third body axis (according to Eq. (2.6). In the third case, it is assumed that actuator failure leads to $100 \%$ degradation in the moment about the third body axis. Therefore, in this case, it is not possible to produce any moment about the third axis.

The maximum control moment $u_{\max }$ that can be produced by healthy actuators is assumed to be $50 \mathrm{Nm}$.

The simulations are carried out in three Sections. In Section 4.1, the actuator failure corresponds to the case three of Table 2. However, no action is taken to compensate for the effect of this failure. In Section 4.2, the actuator fault/failure corresponds to cases two and three of Table 2. The proposed method is employed to compensate for the effect of this fault/failure. Finally in Section 4.3, the robustness of the proposed controller toward uncertainties and disturbances is determined via Monte Carlo simulation.

\subsection{No action is taken to compensate for the effect of actuator failure}

In order to see the consequences of uncompensated faults on the controller performance, the third case of Table 2 (actuator failure) is considered without reconfiguring the controller. The controller coefficients $k_{1}, k_{2}$ and $k_{3}$ are considered to be equal to the initial values presented in Table 1. The response errors corresponding to the uncorrected system are illustrated in Fig. 4. In this figure: $e_{\phi}=\phi-\phi_{r}, e_{\theta}=\theta-\theta_{r}, e_{\psi}=\psi-\psi_{r}$.

According to Fig. $4, e_{\psi}$ has not converged to zero. The reason is contributed to the fact that the control input corresponding to $\psi$ has failed $\left(u_{3}^{\prime}=0\right)$. This figure shows that mission 

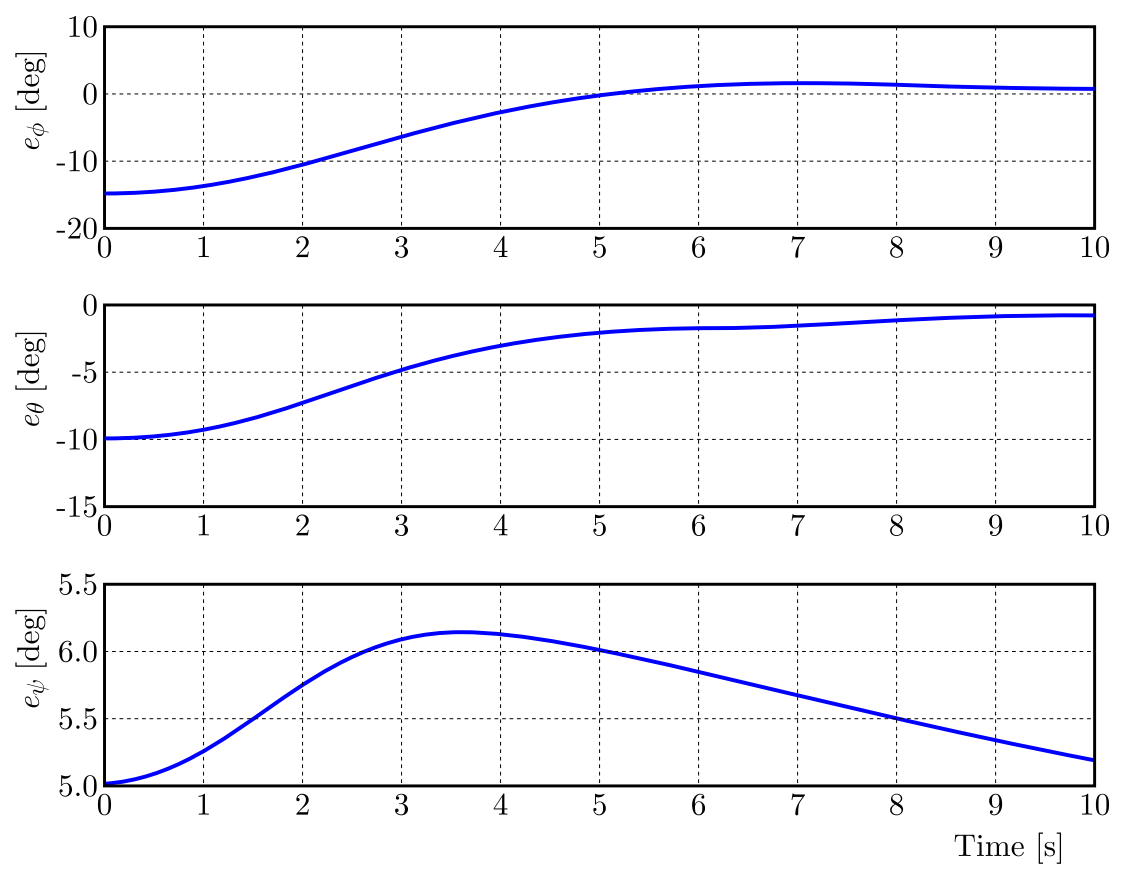

Fig. 4. Response errors (uncompensated system)

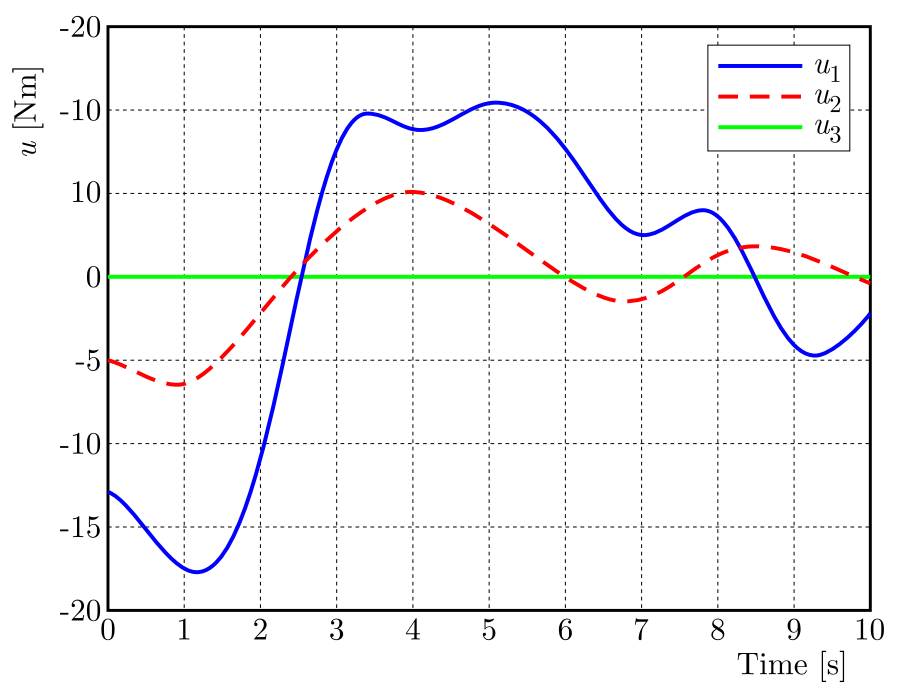

Fig. 5. Control moments (uncompensated system)

objectives will fail if the effect of actuator failure is not compensated. Therefore, at least in this case, system recovery is a necessary task. The control moments are illustrated in Fig. 5.

In comparison to the results obtained in the next Section (Figs. 8 and 9), the control moments are smaller. The reason is simply the fact that the controller does not exploit the available control moments to compensate for the effect of actuator failure.

\subsection{Employing the proposed method to compensate for the effect of actuator fault/failure}

In this case, the proposed AFTC is used to compensate for the effects of faults presented in Table 2. It will be shown that the proposed method has a great effect on the improvement of the faulty system performance.

Figure 6 shows the result of employing the proposed AFTC as three Pareto fronts (corresponding to three scenarios in Table 2). As the faults become larger, the two performance 
measures increase and move to the upper-right corner of the performance space. It should be noted that these Pareto fronts are illustrated for the case $P M_{2}=0$ (the minimal value of $P M_{2}$ ) and, therefore, $P M_{2}$ is not illustrated in Fig. 6.

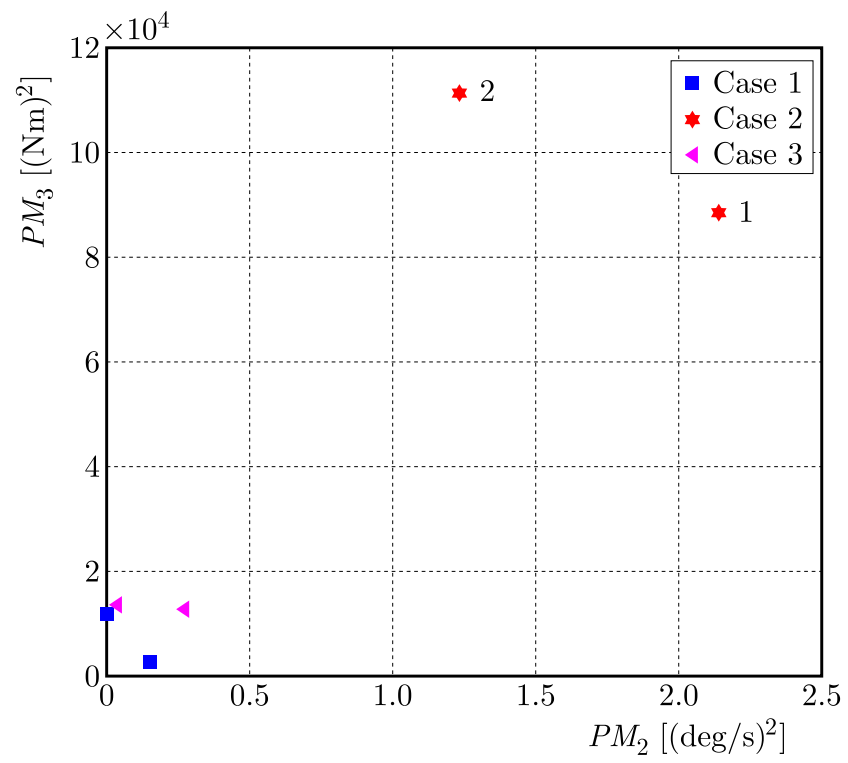

Fig. 6. Pareto fronts for three cases considered in Table 4

In order to show the performance of the controller, the response errors corresponding to the faulty system (design points 1 and 2 in Fig. 6) are illustrated in Fig. 7.
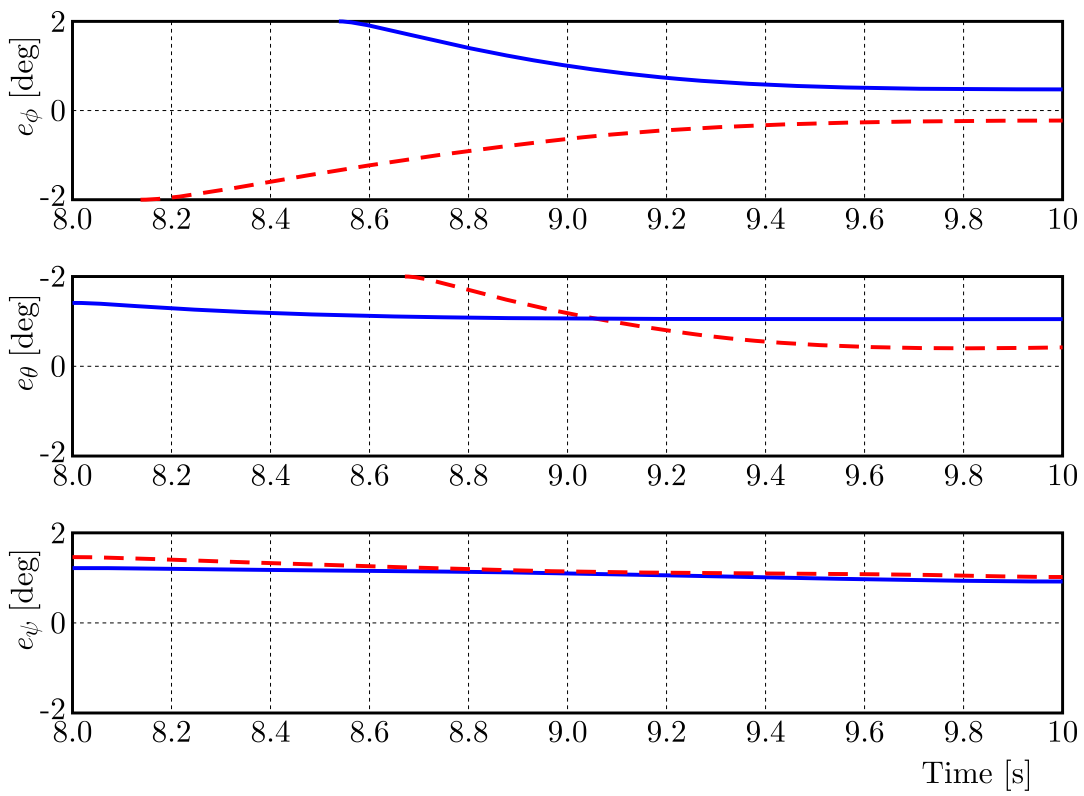

Fig. 7. Response errors (compensated system)

For better comparison, the errors are illustrated for the time interval $[8,10]$ seconds. Compared to Fig. 4, these results show a great improvement in the controller performance. The results also show that the error corresponding to design point 2 is smaller than the error corresponding to design point 1 . As will be seen in the next figures, the energy consumption corresponding to design point 2 is higher than point 1 , which is physically sensible. The corresponding control moments are illustrated in Figs. 8 and 9. 


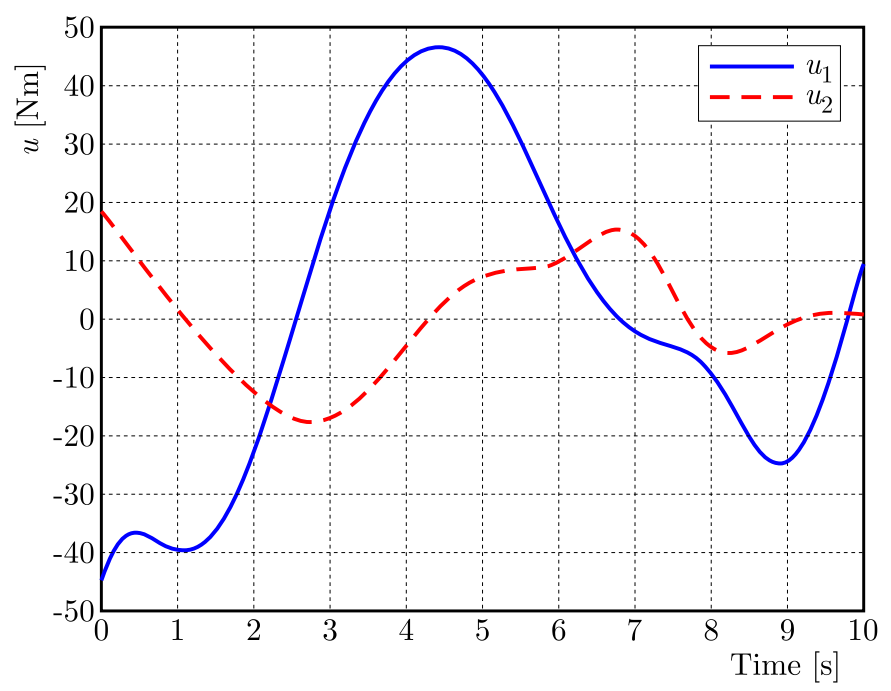

Fig. 8. Control moments (compensated system)

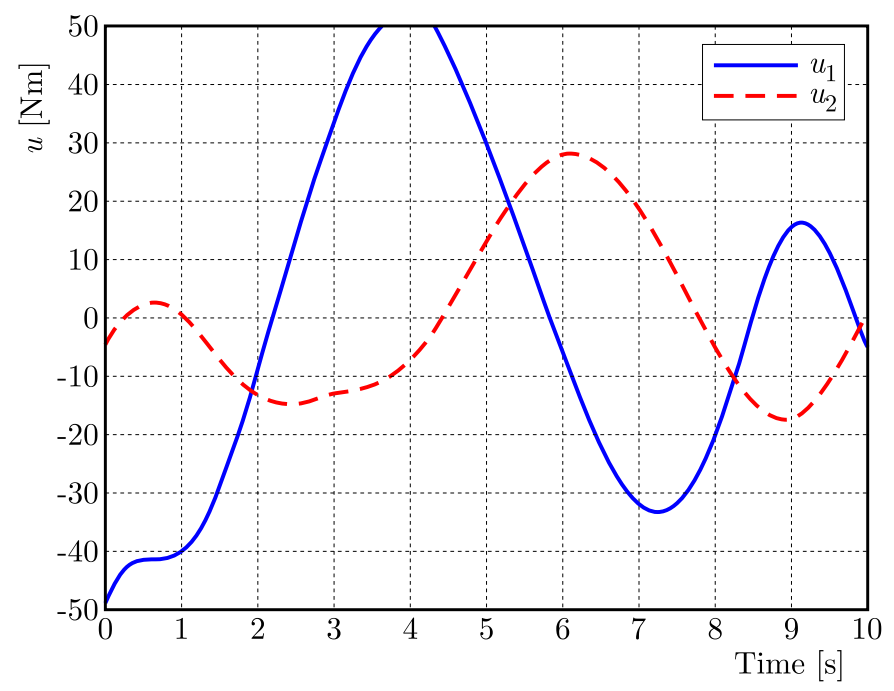

Fig. 9. Control moments (compensated system)

In comparison to Fig. 5, Figs. 8 and 9 show that compensating for the effect of actuator failure requires more energy. Figure 8 corresponds to design point 1 , where the faulty system uses less control effort and, therefore, the controller performance decreases. On the other hand, Fig. 9 corresponds to design point 2, where more energy is used to increase the quality of the response, leading to saturation of the controller.

\subsection{Monte Carlo simulation}

At the end of the simulation Section, a Monte Carlo analysis is presented to analyze the robustness of the proposed controller toward uncertainties and disturbances. Before demonstrating the Monte Carlo results, models of uncertainties and disturbances are explained.

The moment of inertia matrix for the real system is considered as

$$
\mathbf{J}_{\text {real }}=\mathbf{J}+\delta \mathbf{J}=\left[\begin{array}{ccc}
J_{1} & 0 & 0 \\
0 & J_{2} & 0 \\
0 & 0 & J_{3}
\end{array}\right]+\delta \mathbf{J}
$$


where $\delta \mathbf{J}$ is the uncertainty in measuring the moment of the inertia matrix. This parameter is defined according to the following relation

$$
\delta \mathbf{J}=\delta \mathbf{J}_{l b}+\operatorname{rand}\left(\delta \mathbf{J}_{u b}-\delta \mathbf{J}_{l b}\right)
$$

where $\delta \mathbf{J}_{l b}$ and $\delta \mathbf{J}_{u b}$ are the lower and upper bounds of $\delta \mathbf{J}$, respectively. Their values are considered to be $\pm 10 \%$ of $\mathbf{J}$ for diagonal and $\delta \mathbf{J}_{l b}=-10, \delta \mathbf{J}_{u b}=0$ in $\mathrm{kg} \mathrm{m}^{2}$ for non-diagonal elements (engineering orders). The operator "rand" is a random number generator between 0 and 1.

Sensor noise is also considered in the robustness analysis. For this purpose, the noise is considered to be of Gaussian white noise type

$$
w(t)=\frac{1}{\sigma_{N} \sqrt{2 \pi}} \exp \left(-\frac{\left(t-\mu_{N}\right)^{2}}{2 \sigma_{N}^{2}}\right)
$$

where $\mu_{N}$ and $\sigma_{N}$ are the mean and standard deviation, respectively. It is assumed that this noise is added to the angular velocity sensor output, and the sampling time is equal to $0.1 \mathrm{~s}$. $\mu_{N}$ is considered to be zero and $\sigma_{N}$ is assumed to be $0.0001 \mathrm{rad} / \mathrm{s}$.

The disturbance moments are assumed to be sinusoidal functions of time

$$
\mathbf{T}_{d}=\left[\begin{array}{c}
0.01 \sin (0.7 t) \\
0.02 \sin (0.8 t) \\
0.001 \sin (0.1 t)
\end{array}\right] \mathrm{Nm}
$$

which is equivalent to the disturbances in a circular orbit with $400 \mathrm{~km}$ height, for the considered spacecraft (Wang et al., 2013).

50 simulations have been carried out to perform the Monte Carlo analysis. The error of Euler angles $e_{\phi}=\phi-\phi_{r}, e_{\theta}=\theta-\theta_{r}, e_{\psi}=\psi-\psi_{r}$ at $t=t_{f}$ are obtained for every simulation and then, their mean and standard deviation are calculated. According to these values, the normal probability density function is calculated according to the following equation

$$
\mathbf{P}=f(\mathbf{x} \mid \mu, \sigma)=\frac{1}{\sigma \sqrt{2 \pi}} \exp \left(\frac{-(\mathbf{x}-\mu)^{2}}{2 \sigma^{2}}\right)
$$

In this equation, the vectors $\mathbf{x}$ and $\mathbf{P}$ are the final values of Euler angle errors and the corresponding probability distribution, respectively.

The robustness of controller in design point 2 (Fig. 6) is considered and the probability distributions for the final values of Euler angle errors are illustrated in Fig. 10. According to Fig. 10, the mean value of $e_{\psi}$ at $t=t_{f}$ is 1 degree, which is larger than the mean values of $e_{\phi}$ and $e_{\theta}$ at $t=t_{f}$.

Finally, it should be reminded that pure control moments have been considered in the controller design. The selection of actuation system is another topic that should be considered separately.

\section{Conclusion}

An active attitude fault tolerant controller was proposed for the attitude control problem. The attitude control problem is of importance in many space-based applications. Severe actuator fault/failure and finite time convergence were especially emphasized as two important challenges. These two challenges, especially the first one, are important. This is due to the fact that the occurrence of fault/failure degrades the performance of the controller. Lyapunov's direct method and a theorem were used to resolve these challenges to some extent. Simulation results demonstrated the capabilities of the proposed controller to make the faulty system response converge to the reference attitude. 

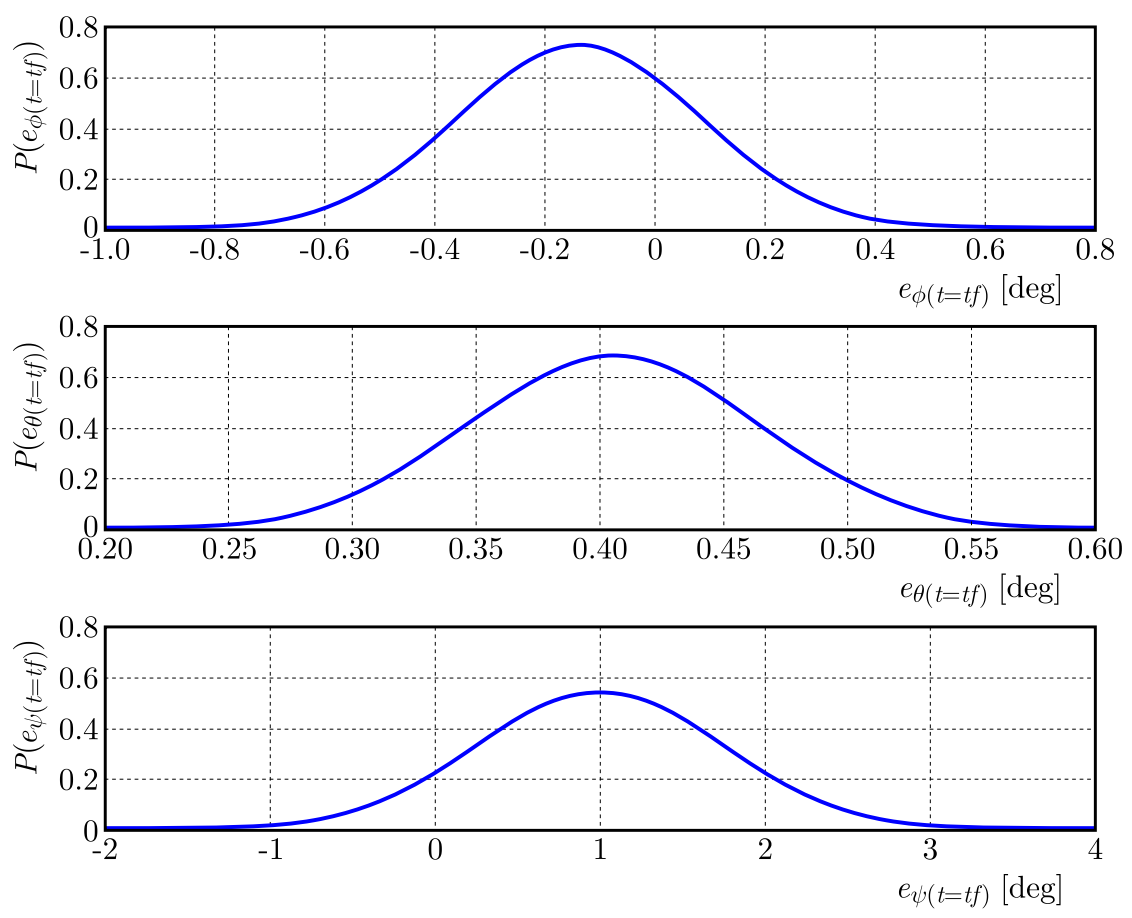

Fig. 10. Probability distributions for design point 2

\section{References}

1. Alikhani A., 2017, Passive fault-tolerant control of an underactuated re-entry capsule, Journal of Aerospace Technology and Management, 9, 4, 442-452, DOI: 10.5028/jatm.v9i4.771

2. Benosman M., Lum K.Y., 2009, Online reference reshaping and control reallocation for nonlinear fault tolerant control, IEEE Transactions on Control Systems Technology, 17, 2, 366-379, DOI: 10.1109/TCST.2008.2000983

3. Blanke M., Kinnaert M., Lunze J., Staroswiecki M., 2006, Diagnosis and Fault-Tolerant Control, Springer, DOI: 10.1007/978-3-540-35653-0

4. De Boor C., 1978, A Practical Guide to Splines, Hardcover, ISBN: 978-0-387-95366-3

5. FEKIH A., 2014, Fault diagnosis and fault tolerant control design for aerospace systems: a bibliographical review, American Control Conference (ACC), DOI: 10.1109/ACC.2014.6859271

6. Hu Q., Niu G., WANG C., 2017, Spacecraft attitude fault-tolerant control based on iterative learning observer and control allocation, Aerospace Science and Technology, 75, April, 245-253, DOI: $10.1016 /$ j.ast.2017.12.031

7. Hu Q., Shao X., Guo L., 2018, Adaptive fault-tolerant attitude tracking control of spacecraft with prescribed performance, IEEE/ASME Transactions on Mechatronics, 23, 1, 331-341, DOI: 10.1109/TMECH.2017.2775626

8. JIANG J., YU X., 2012, Fault tolerant control systems: a comparative study between active and passive approaches, Annual Reviews in Control, 36, 1, 6072, DOI: 10.1016/j.arcontrol.2012.03.005

9. JiAnG J., ZHANG Y., 2006, Accepting performance degradation in fault tolerant system design, IEEE Transactions on Control Systems Technology, 14, 2, 284-292, DOI: 10.1109/TCST.2005.860515

10. Khalil H.K., 2014, Nonlinear Control, Pearson, ISBN-13: 978-0133499261

11. Linlin L., 2016, Fault Detection and Fault-Tolerant Control for Nonlinear Systems, Springer, DOI: 10.1007/978-3-658-13020-6 
12. Lunze J., Richter J.H., 2008, Reconfigurable fault-tolerant control: a tutorial introduction, European Journal of Control, 14, 5, 359-386, DOI: 10.3166/ejc.14.359-386

13. Marler R.T., Arora J.S., 2004, Survey of multi-objective optimization methods for engineering, Structural and Multidisciplinary Optimization, 26, 369395, DOI: 10.1007/s00158-003-0368-6

14. Miksch T., Gambier A., 2011, Fault-tolerant control by using lexicographic multi-objective optimization, Proceedings of 8th Asian Control Conference (ASCC), Kaohsiung, Taiwan, May 15-18

15. Moradi R., Jegarkandi M.F., Alikhani A., 2017, A heuristic active fault tolerant controller for the stabilization of spacecraft, Acta Polytechnica Hungarica, 14, 6

16. Noura H., Theilliol D., Ponsart J.C., Chamseddine A., 2009, Fault Tolerant Control Systems Design and Practical Applications, Springer-Verlag, London, DOI: 10.1007/978-1-84882-653-3

17. TAfazoli, M., 2008, A study of on-orbit spacecraft failures, Acta Astronautica, 64, 23, 195-205, DOI: 10.1016/j.actaastro.2008.07.019

18. Wang D., Jia Y., Jin L., Xu S., 2013, Control analysis of an underactuated spacecraft under disturbance, Acta Astronautica, 83, 44-53, DOI: 10.1016/j.actaastro.2012.10.029

19. WitcZAK M., 2014, Fault Diagnosis and Fault-Tolerant Control Strategies for Non-Linear Systems, Springer, DOI: 10.1007/978-3-319-03014-2

20. Yin S., Xiao B., Ding S., Zhou D., 2016, A review on recent development of spacecraft attitude fault tolerant control system, IEEE Transactions on Industrial Electronics, 63, 5, 3311-3320, DOI: 10.1109/TIE.2016.2530789

21. Zhang Y., JiAng J., 2008, Bibliographical review on reconfigurable fault-tolerant control, Annual Reviews in Control, 32, 2, 229-252, DOI: 10.1016/j.arcontrol.2008.03.008

22. ZiPfel P.H., 2007, Modeling and Simulation of Aerospace Vehicle Dynamics, AIAA Education Series, 2nd Ed., DOI: 10.2514/4.862182 